\title{
Idiopathic Pneumoperitoneum in A Pediatric Patient: A Case Report
}

\author{
Lorena Míguez Fortes ${ }^{1}$, Miriam García González ${ }^{1}$, Noelia Curros Mata ${ }^{1}$, \\ Isabel Casal Beloy ${ }^{1}$, Isabel Suárez Dono ${ }^{2}$, Iván Somoza Argibay ${ }^{1}$ \\ ${ }^{1}$ Departament of Pediatric Surgery \\ ${ }^{2}$ Departament of Radiology
}

Children’s Hospital Teresa Herrera (Complejo Hospitalario Universitario de A Coruña), Spain

\begin{abstract}
Pneumoperitoneum is consequence of an intra-abdominal visceral perforation and requires emergent laparotomy in most of cases. However had been described in adults and children causes of spontaneous pneumoperitoneum and some of them could be treated with a conservative approach. We present the case of a 7 month child who presents radiologic findings of pneumoperitoneum, fever, leukocytosis and elevation of acute phase reactants. We performed a laparotomy and no evidence of perforation or other causes of pneumoperitoneum were found.
\end{abstract}

Keywords: Pneumoperitoneum, Spontaneus pneumoperitoneum, Idiopathic pneumoperitoneum, Children

Abbreviations

SP: Spontaneous pneumoperitoneum, CRP: C Reactive Protein, PCI: Pneumatosis Cystoides Intestinalis

\section{INTRODUCTION}

Pneumoperitoneum is a radiologic term denoting an abnormal collection of air in the peritoneal cavity. This condition reflects visceral perforation that requires urgent surgical exploration and intervention in 85 to $95 \%$ of the cases [1-3]. In the appropiate clinical setting, the radiographic presence of intraperitoneal air is considered a diagnostic finding of perforation [4]. However, pneumoperitoneum without evidence of a perforated viscus has been reported [3,5]. This is called spontaneous penumoperitoneum or "non-surgical" pneumoperitoneum [6]. Nonsurgical pneumoperitoneum can be a consequence from several causes or can be idiopathic. [7].

Spontaneous pneumoperitoneum is characterized by a benign course and it can be managed conservatively $[8,9]$.

Cases of non-surgical pneumoperitoneum have been well documented in adults but in pediatric population evidence has been limited. However there are recent studies in children of spontaneus pneumoperitoneum managed non-surgically [10].

This supose a significant management dilemma for surgeons, especially when signs of peritonitis are absents or when the cause is unknown before laparotomy and above all when we are talking about pediatric population.

\section{CASE REPORT}

We report the case of a 7-month-old patient with a 3 days history of fever. She had a medical history of partial agenesis of the corpus callosum and a psychomotor retardation. She was being studied by her pediatrician for a suspected immunodeficiency. The cause of this suspicion was the periodic fever.

She presented a 3 days history of fever $\left(38.8^{\circ} \mathrm{C}\right)$ and partial rejection of ingestion and irritability. The patient was febrile in the emergency department and physical examination reveals pale skin and rigth painful abdomen but no defense or any peritoneal irritation sign. Laboratory examination presented an elevated white blood cell count, neutrophilia and a CRP of $30 \mathrm{mg} / \mathrm{Dl}$ (reference range 0 - 
1). Free abdominal fluid with detritus was observed in abdominal ultrasound, so abdominal focus was suspected.

Due to abscense or peritoneal irritation sings the patient was observed with improvement of clinical status and a normal, soft and non-painful abdomen.

An abdominal radiography was performed and showed pneumoperitoneum with large amount of intraperitoneal free air that draws the wall of thin handles and the peritoneal folds surrounding liver and spleen.

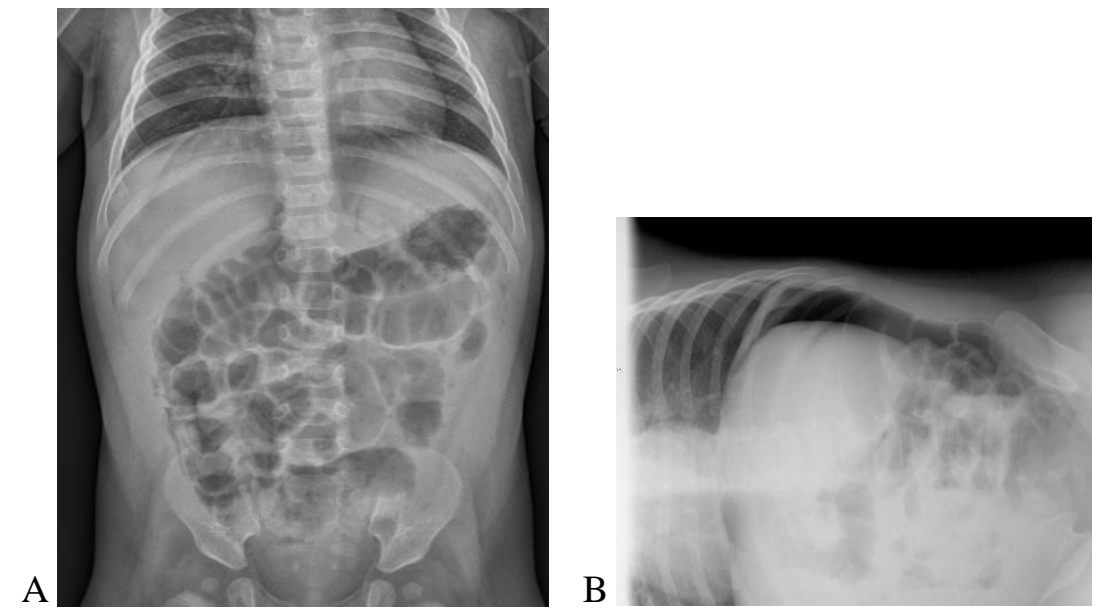

Figure $1(\mathbf{A}, \mathbf{B})$ - Figure A shows a supine abdominal films thats reveals a change in liver density (arrow) provocated for free peritoneal air. The free air draws the wall of thin handles and the peritoneal folds surrounding liver and spleen(arrow).

Figure $\mathbf{B}$ is a decubito film (left side down) that shows a masive amount of air (arrow) within the abdominal caivity.

Due to the radiologic findings, analytical dates and fever a perforation was suspected and we performed and emergent laparotomy. A moderate amount of free intra-abdominal fluid was observed. The small bowel and colon were examined but no evidence of perforation was found. The abdominal cavity was filled with normal saline and air was infused through the nasogastric tube into de stomach but no air leakage from the GI tract was noted. Finally because no cause of the pneumoperitoneum had been found we performed prophylactic appendicectomy.

The postoperative course was uneventfully: she was treated for 7 days with triple antibiotic therapy (cefotaxime, gentamicin and metronidazole) with a prompt recovery. Laboratory values became normal with CRP decline from 30 to $3 \mathrm{mg} / \mathrm{dL}$ and standardization of white cell recount. She was without fever and oral tolerance was iniciated at 2 th postoperative day with good clinical evolution. The patient was discharged at 7 postoperative day.

Cultures of the peritoneal fluid were negative and the pathological anatomy of the normal appendix was negative.

\section{DISCUSSION}

Pneumoperitoneum is a radiologic term denoting an abnormal collection of air in the peritoneal cavity. Most of the cases it is due to intra-abdominal viscus perforation and requires emergent surgical exploration. Radiographic evidence of free intraperitoneal air was linked to intra-abdominal disease in the early 1900s and continues to be an useful diagnostic tool for perforation of intraabdominal viscus. [11]

In fact it has been reported that pneumoperitoneum results from visceral perforation in $85-95 \%$ of cases [1-3]. When a new onset of pneumoperitoneum is present in a patient with fever, abdominal pain, leukocytosis, and signs of peritoneal irritation, surgical management is indicated. [7]

There is however a $5-15 \%$ of patients with radiologic findings of pneumoperitoneum that are not result of a perforation [3,5,12], giving rase the concept of "non-surgical" or spontaneous pneumoperitoneum. This is defined as abnormal collection of air in the peritoneal cavity that could be 
managed with observation and supportive care succesfully [14]. Often, patients with this disorder undergo laparotomy with no intraoperative evidence of visceral disease. This concept have been well documented in adults but in children there are only a few reports that document intances of spontaneous pneumoperitoneum managed by a conservative approach. [13, 14]

It has been suggested that the cause of pneumoperitoneum and the clinical sings determine the choice of surgical or conservative treatment. When sings and symptoms of "acute abdomen" are present, surgical management is mandatory. But in cases of non-surgical pneumoperitoneum with mild symptoms and without sings of peritoneal irritation we can try conservative treatment. [9] In our case, the patient presented pneumoperitoneum with fever and leukocytosis that is why surgical exploration was indicated.

Spontaneum pneumoperitoneum is well documented and associated with numerous causes as intrathoracic, intraabdominal, gynecologic, iatrogenic and other miscellaneous causes.[8]

Pneumoperitoneum can be a complication of other abdominal procedements: abdominal surgery, peritoneal dialysis and endoscopic gastrointestinal procedures. Its occurs after open abdominal surgery in $60 \%$ of cases and after laparoscopic procedures in about $25 \%$. Progressive resolution of free air is expeted within 5 days in $97 \%$ of cases [15]

Pneumatosis cystoides intestinalis (PCI) in pediatric population can be the first sign of underlying diseases such as necrotizing enterocolitis, peptic ulcers, pyloric obstruction, intestinal obstruction, inflammatory bowel disease and connective tissue disease and is associated with SP without perforation [16]. PCI is characterized by intramural gas-filled cysts wich rupture causes pneumoperitoneum and generally resolves spontaneusly. [17]

Thoracic causes include trauma (also barotraumas), pneumothorax or cardiopulmonary resucitation. In the pediatric population, nonsurgical pneumoperitoneum occurs in 1 to $3 \%$ of mechanically ventilated preterm infants, depending of the mode of ventilation. [7]

Gynecologic causes are seen less frequently in children and can be resulted of a pelvic inflammatory disease or can be a sign of sexual abuse. [10]

Finally, there exists a group of patients in whom there are no demonstrable causes for the development of pneumoperitoneum and many of these cases have a negative laparotomy. These are true cases of idiopathic pneumoperitoneum. Is was only after a negative laparotomy and no relevant history or radiological findings that these cases can be asumed to be idiopathic. [19,20]

In our patient, we did not found positive findings like perforation evidence or positive peritoneal cultures so diagnosis of idiopathic pneumoperitoneum is appropiate.

It has been proposed in idiopathic pneumoperitoneum, especially in the presence of a "negative laparotomy" that a subclinical microperforated hollow viscus may be present. [21,22]. As in our patient pneumoperitoneum may have evolved from a subclinical perforation or be due to other factor that remain enigmatic.

Its is widely described that conservative management of idiopathic pneumoperitoneum is indicated in abscense of peritoneal signs, leukocytosis, fever and abdominal pain and distension. Idiopathic pneumoperitoneum is a diagnostic and terapeuthic challenge. When some of these signs are present it is indicated to perform a laparotomy and in fact this is the only way to definitively diagnose this entity after ruling out the remaining possibles causes of pneumoperitoneum.

\section{Conclusions}

Radiographic evidence of pneumoperitoneum is considered an perforation sign, but is widely described that in the absence of peritoneal signs, fever, leukocytosis, significant abdominal pain, distension, or clinical deterioration, non-operative management should be considered. Causes of sponateneous pneumoperitoneum are reported in literature but diagnosis and management of idiopathic pneumoperitoneum represents a great challenge for the surgeon. We must keep it on mind in pediatric surgery and to orient our therapeutic attitude according to the possible causes, clinical symptoms and suplementary tests. 


\section{REFERENCES}

[1] McGlone FB, Vivion CG, Meir L: Spontaneous pneumoperitoneum. Gastroenterology; 51:393398 (1966)

[2] Roh JJ, Thompson JS, Harned RK, et al: Value of pneumoperitoneum in the diagnosis of visceral perforation. Am J Surg 146:830-833 (1983)

[3] Winek TG, Mosely HS, Grout G, et al: Pneumoperitoneum and its association with ruptured abdominal viscus. Arch Surg 123:709-712 (1988)

[4] Sabiston DC: Textbook of Surgery. 14th Edition. Philadelphia, PA, Saunders (1991)

[5] C. Gantt, W. Daniel, G. Hallenbeck, Nonsurgical pneumoperitoneum, Am. J. Surg. 134:411-414 (1977)

[6] F. McGlone, C. Vivion, L. Meir, Spontaneous pneumoperitoneum, Gastroenterology 51: 393398 (1966)

[7] Richard A. Mularski, MD; Jeffrey M. Sippel, MD, MPH; Molly L. Osborne, MD Phd, Pneumoperitoneum: A review of non surgical cases, Crit Care Med Vol. 28 No.7 (2000)

[8] Mularski RA, Ciccolo ML, Rappaport WD: Non surgical causes of pneumoperitoneum. West J Med 170:41-46

[9] Karaman A, Demirbilek S, Akin M, Gürünlüoglu K, Irsi C: Does pneumoperitoneum always requiste laparotomy? Report of six cases and review of literature, Pediatr Surg Int 2005, 21:819824

[10] Caitlin Tallant, Aaron Tallant, Jason Nirgiotis, Janet Meller, Spontaneous pneumoperitoneum in pediatric patients: A case series, International Journal of Surgery Case Reports 22:55-58 (2016)

[11] Dandy WE, Pneumoperitoneum. Ann Surg 70:378-383 (1916)

[12] R. Freeark, J. Raffensper, J. Condon, Pneumperitoneum in infancy, Surg Gynecolog. Obstet 113:623-630 (1961)

[13] Porter A. Spontaneous pneumoperitoneum in the newborn. New Engal J Med 254:694-696 (1956)

[14] Zerella JT, McCullough JY. Pneumoperitoneum in infants without gastrointestinal perforation. Surgery 89:163-167 (1981)

[15] Stapakis JC, Thickman D: Diagnosis of pneumoperitoneum: Abdominal TC vs upright chest film. J Comput Assist Tomogr, 16:713-716 (1992)

[16] M. Alassaf, Recurring spontaneous aseptic pneumoperitoneum presenting secondary to an unrelated chief compliant: a case report. Int J. Surg. Case Rep. 7:96-98 (2014)

[17] Longo WE, Ballantyne GH, Graham AJ: Pneumatosis cystoides intestinalis, J Clin Gastroenterol, 9:571-573 (1987)

[18] NMA Williams, DFL Watkin, Spontaneous pneumoperitoneum and other nonsurgical causes of intraperitoneal free gas, Postgrad Med J, 73:531-537 (1997)

[19] Hinckel CL. Spontaneous pneumoperitoneum without demonstrable visceral perforation. AJR, 43:377-382 (1940)

[20] Leys D. Pneumoperitoneum. BMJ ii:562 (1944)

[21] Simpson AJ. Spontaneous asymptomatic pneumoperitoneum secondary to jejunal diverticulosis. J Med Soc N J,73:1065-1067 (1976)

[22] Hoover EL, Cole GD, Mitchel LS, Adams CZ Jr, Hasset J. Avoiding laparotomy in nonsurgical pneumoperitoneum. Am J Surg, 164:99-103 (1992) 